\title{
All forefoot's pain are not morton's neuroma-a pictorial review of the forefoot musculoskeletal conditions mimicking mortons neuroma
}

\begin{abstract}
Introduction: Ultrasound (US) and Magnetic resonance imaging (MRI) of the foot are often requested by foot and ankle specialists or general physicians (GPs), and the reason for these referral requests to Musculoskeletal Radiologists, is usually forefoot and midfoot pain. The request card for these patients usually states Morton's neuroma as a working/suspected diagnosis. However there are many other conditions that can mimic Morton's neuroma.
\end{abstract}

Materials and methods: This article demonstrates multiple conditions that we have come across in our practice. Between July 2016 and February 2017 we received 173 consecutive patients from GP and specialists for ultrasound of foot with various clinical suspicions. Standard ultrasonography of the foot was performed using high frequency probes by consultant muskuloskeltal radiologists.

Results: Various differential diagnoses included intermetatarsal bursitis, stress frature, degenerative changes of the metatarsophalangeal and tarsometatarsal joints, inflammatory arthropathy causing synovitis and joint effusions, turf toe, sesamoiditis, freiberg's disease, plantar plate disruption, tenosynovitis of flexor or extensor tendons. Additionally benign conditions have been detected at such as soft tissue disorders, ganglions, calluses, adventitial bursitis, plantar fibromatosis and haemangioma. Furthermore malignant conditions such as giant cell tomour of tendon sheath and schwannomas were also detected.

Conclusion: To improve the diagnosis of forefoot/ non-specific foot pain, healthcare professionals need to collect a complete and careful history, perform thorough physical examinations and to be more aware about the possible conditions that lead to foorfoot/foot pain. This will avoid unnecessary visits to GPs, orthopaedic surgeons and radiologists.

Keywords: foot pain, forefoot pain, morton's neuroma, ultrasound
Volume 5 Issue 5 - 2018

\author{
A Mehta, ${ }^{\prime}$ A Chourasia, ${ }^{2} \mathrm{C}$ Urigo, ${ }^{3}$ A Sahu ${ }^{3}$ \\ 'Poznan University of Medical Sciences, Poland \\ ${ }^{2}$ Department of Trauma and Orthopaedics, South Warwickshire \\ NHS Foundation Trust, Poland \\ ${ }^{3}$ Ealing Hospital Radiology Department, London Northwest \\ University Healthcare Trust, Poland
}

Correspondence: Aanchal Mehta, Department of Medicine (MD), Poznan University of Medical Sciences, Email Aanchal.mehta 18@imperial.ac.uk

Received: July 3I, 2018 | Published: September 25, 2018
Abbreviations: US, ultrasound; MTP, metatarsophalangeal; AVM, arteriovenous malformation; GP, general physician

\section{Introduction}

Forefoot pain is a common clinical finding and is often poorly understood. Clinically forefoot pain is termed metatarsalgia as it is most often localised in the metatarsals and in the surrounding soft tissues. Several disorders are associated with forefoot pain and they can can be traumatic or non-traumatic such as Freiberg infarction, Morton's neuroma, bursitis, sesamoid disorders and stress fractures of metatarsals, tendinopathies, arthropathies, infection and even neoplasms. Since these conditions can often mimic each other, to yield a specific diagnosis, a combination of detailed medical history, physical examination and different imaging techniques are used..$^{1-3}$

\section{Morton's neuroma}

Many of the forefoot's pain are usually labeled as Morton's neuroma (or Morton's metatarsalgia, Morton's neuralgia, and plantar neuroma).
Morton's neuroma is a benign condition affecting predominantly the second and third intermetatarsal spaces, most commonly caused by inflammation/scarring of an intermetatarsal plantar nerve. This condition most often presents in middle-aged females, who complain of burning and tingling sensation in the second and third intermetatarsal spaces. This pain is made worse by wearing high-heeled shoes with a narrow toe space and relieved upon shoe removal. This condition was recognized as a separate entity in 1876, by an American orthopedic surgeon, Thomas George Morton. ${ }^{4}$

A careful medical history, physical examination and the correct imaging techniques are needed to assert the set of characteristics that define Morton's neuroma. The physician must preclude the differential diagnoses of Morton's neuroma such as bursitis, stress fractures, MTP joint pathologies, fibromyalgia, rheumatoid arthritis associated conditions (ie rheumatoid nodules/synovitis) and other chronic pain disorders that affect the forefoot. ${ }^{5}$ Techniques such as physical examination and history can often mislead resulting in an erroneous conclusion, therefore imaging is one way by which a specific diagnosis can be made. 


\section{Ultrasound (US)}

An extensively used tool aimed to screen the forefoot and its pathologies is represented by the ultrasound scan. Both dorsal and plantar angles can be visualized using this technique that can also classify these lesions as cystic or solid. Moreover to assess the biological nature of the lesion, colour and pulsed Doppler effects can be added. US is widely used in the diagnosis of Morton's neuroma and its differential diagnostic conditions such as intermetatarsal bursitis. ${ }^{6}$ In terms of Morton's neuroma, US has been used to guide percutaneous treatments. ${ }^{7}$ Additionally, US can be used to ascertain the integrity and structure of the tendons of the forefoot and the plantar plate. ${ }^{8} \mathrm{~A}$ few years ago, US was also used in extraction of foreign bodies as well as to detect and localize metatarsal fractures that were otherwise negative on radiographs. ${ }^{9,10}$

The aim of this article is to highlight conditions that we usually encountered in our practice that can mimic/present like Morton's neuroma. With the help of ultrasound we can differentiate such ailments and prove that not all forefoot's pain are Morton's neuroma.

\section{Materials and methods}

173 patients were examined between July 2016 and February 2017 at Ealing hospital site of London Northwest Healthcare University NHS Trust. These have been referred by the GPs or specialists for ultrasound of the foot for various specific and non-specific forefoot pain. These patients underwent standard ultrasonography of the foot using a high frequency probes.

\section{Results and discussion}

Out of 173 patients, the majority presented with unilaterial right sided forefoot issues $93 / 173(53.7 \%)$. The second most commonly presented subset was the left foot with 68/173(39.3\%) cases. A small set of patients presented with bilateral foot referrals of 12/173(6.9\%) as seen in Figure 1 below.

Out of the 173 referrals in $107(61.8 \%)$ cases the most common symptom was pain, $12(6.9 \%)$ were referred as swelling, while a small subset of others had burning sensations, pins \& needles and odd sensations. These have been summarised in Figure 2 below.

The most common ultrasound finding was Intermetatarsal bursitis in $51(29.5 \%)$ cases, followed by Morton's Neuroma in $41(23.7 \%)$ cases, degenerative changes of joints in 11(6.3\%) cases, while in small group of patients the findings were of ganglion cysts, adventitial bursitis, tenosynovitis, fibroma, lipoma, schwannoma and arteriovenous malformations. A summary of this has been presented in Figure 3 below.

Figure 4-15 show a range of ultrasound findings obtained from our patients group. These images highlight the ultrasound features of various conditions that can mimic Morton's neuroma. While the presenting symtoms may be very suggestive of Morton's neuroma, these images prove that there are multiple disorders that should not be confused with Morton's neuromas and have their own peculiar ultrasound features.

Considering the images above, while the clinical symptoms may be suggestive of a Morton's neuroma, there are abundant other conditions to take into consideration.
Forefoot disorders represent a significant source of pain and discomfort to patients and therefore a careful clinical history taking and a focused physical examination is the key to identify a plausible cause of the pain.

Once a cause is identified and confirmed by an imaging technique such as an ultrasound scan, the correct treatment options can be instituted.

In case of Morton's neuroma, a number of conservative and non conservative options are used, such as shoe modifications, corticosteroid injections and appliances.

Surgical intervention should be considered if the all conservative measures are unsuccessful and an appropriate referral to foot and ankle specialists should be made.

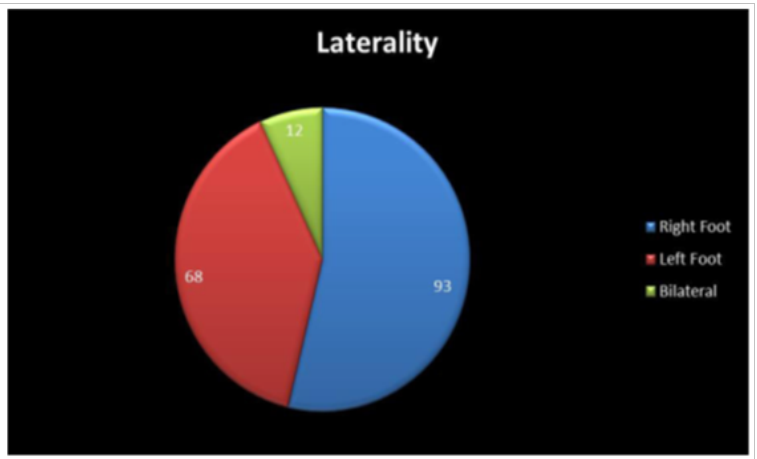

Figure I A pie chart showing laterality of presenting symptoms and subsequent scans.

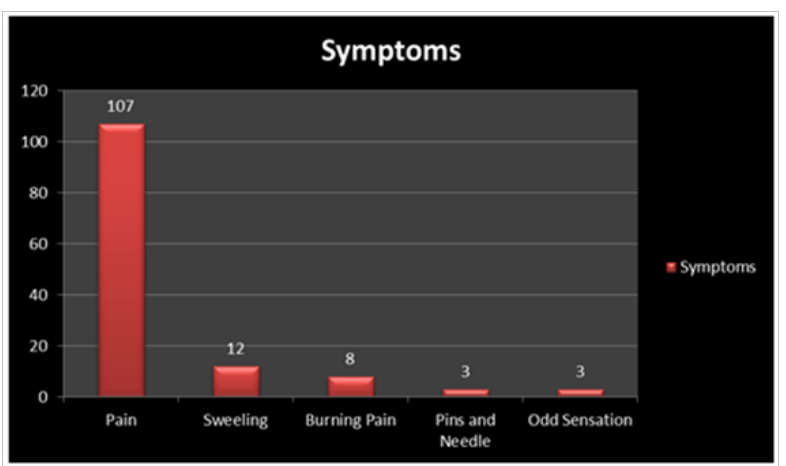

Figure $2 \mathrm{~A}$ bar graph to show different symptoms presented by our patient group.

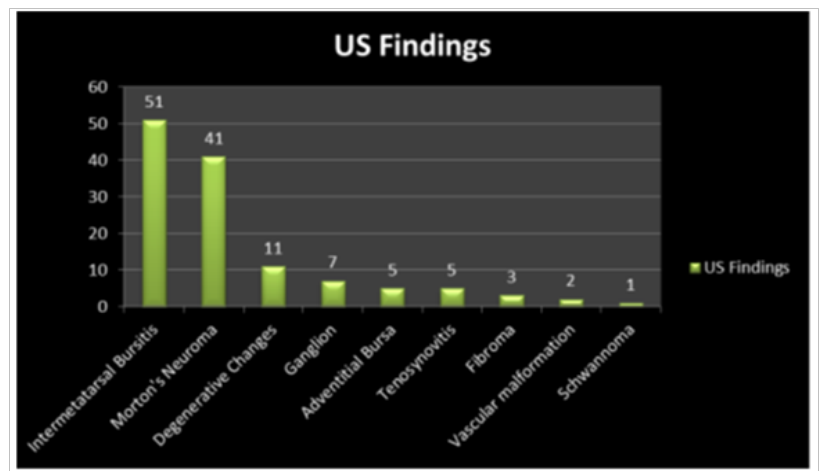

Figure $3 \mathrm{~A}$ bar graph to show the various differential diagnoses concluded based on US findings. 


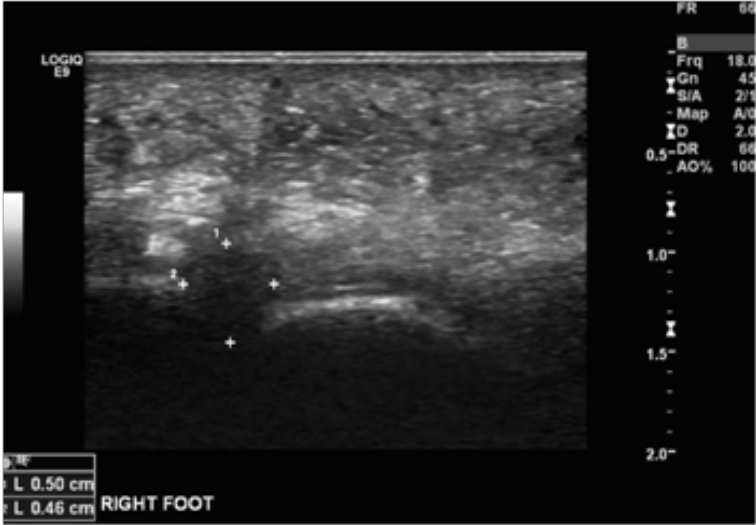

Figure 4 US image of Morton's neuroma.

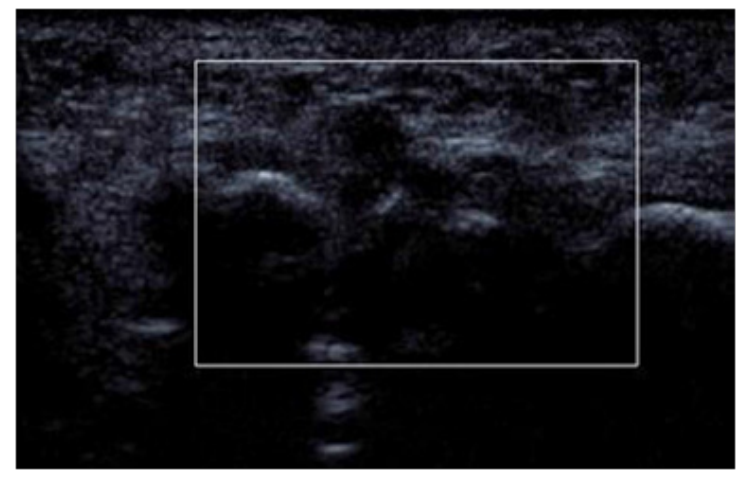

Figure 5 US image of intermetatarsal bursitis.

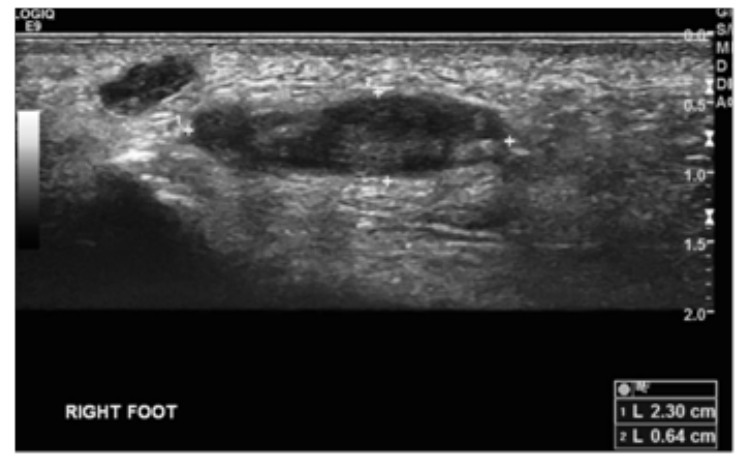

Figure 6 US image shows an atypical fibromatous lumps/keratinous epidermal nodules.

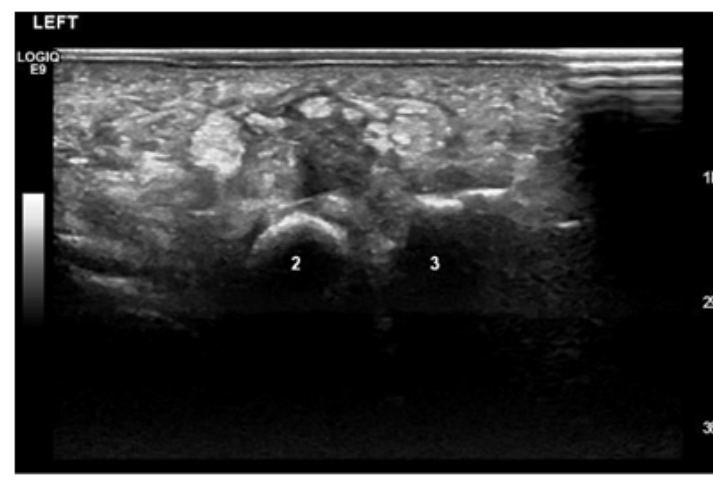

Figure 7 US Image shows an adventitial bursa.

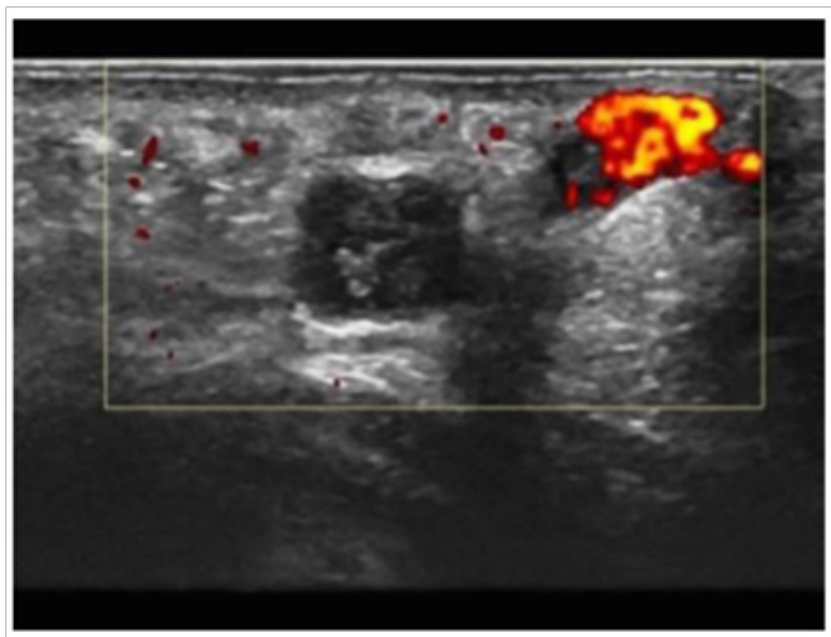

Figure 8 US image with power doppler shows a vascular lesion (suspected AVM).

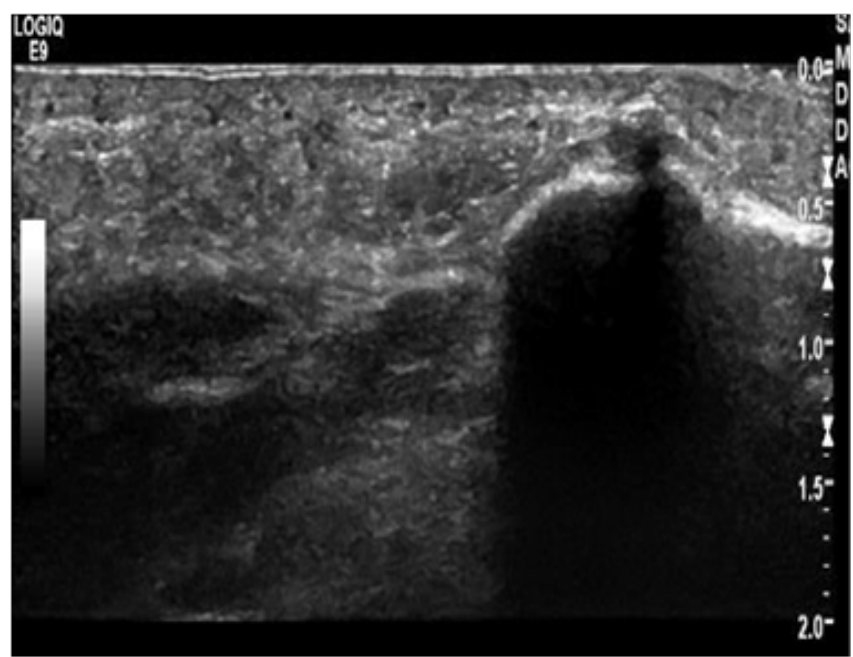

Figure 9 US image shows a small exostosis.

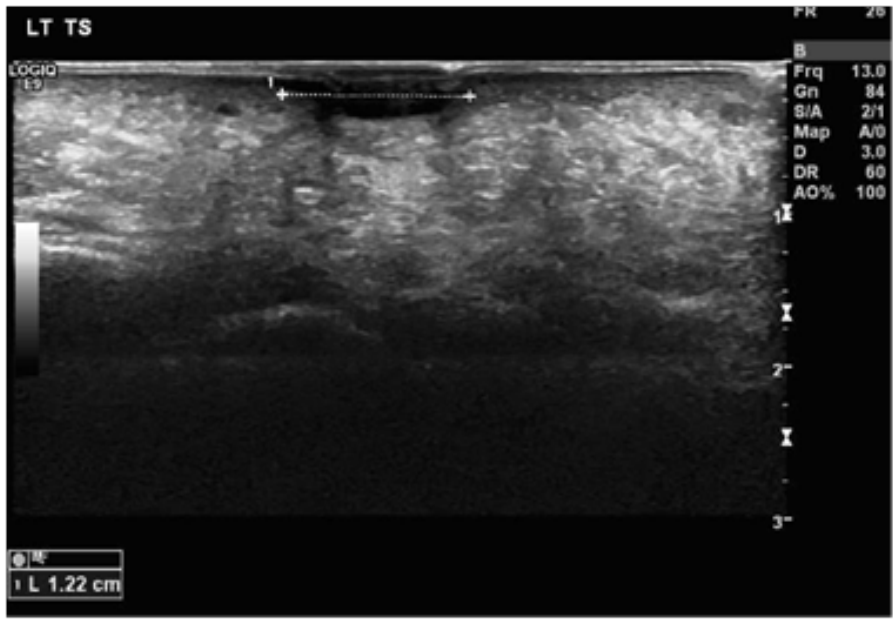

Figure 10 US image shows a corn, callus or verruca.

Citation: Mehta A, Chourasia A, Urigo C, et al.All forefoot's pain are not morton's neuroma-a pictorial review of the forefoot musculoskeletal conditions mimicking mortons neuroma. Int J Radiol Radiat Ther. 2018;5(5):280-284. DOI: 10.15406/ijrrt.2018.05.00179 


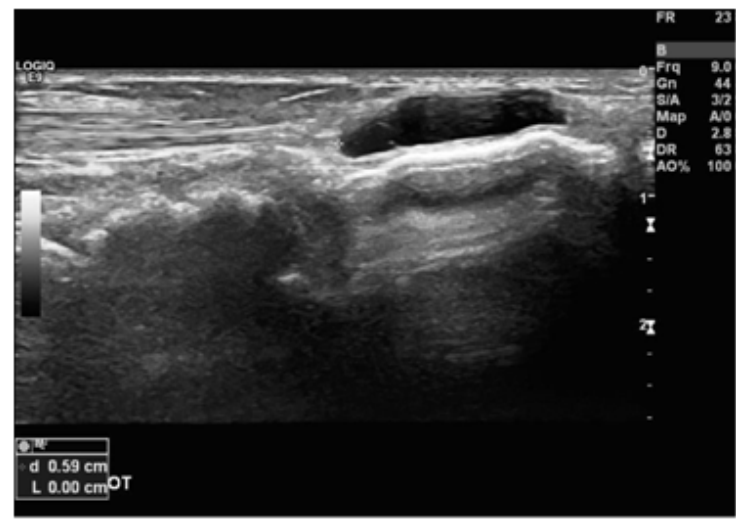

Figure I I US Image shows a ganglion cyst at the TMT joint.

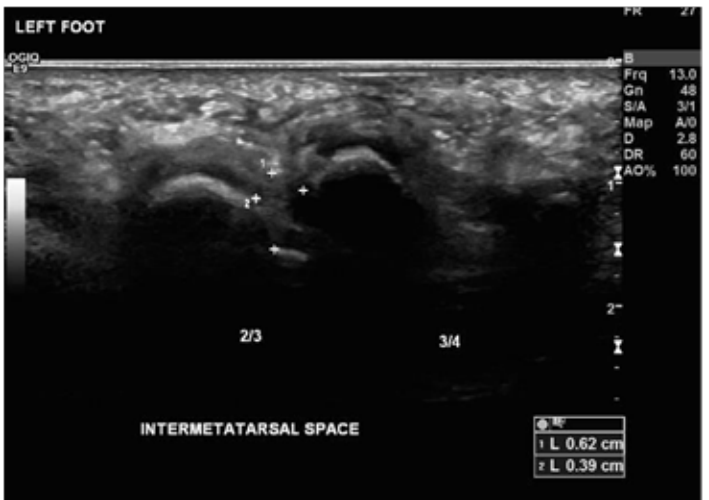

Figure I 2 US image shows a neurobursal complex.

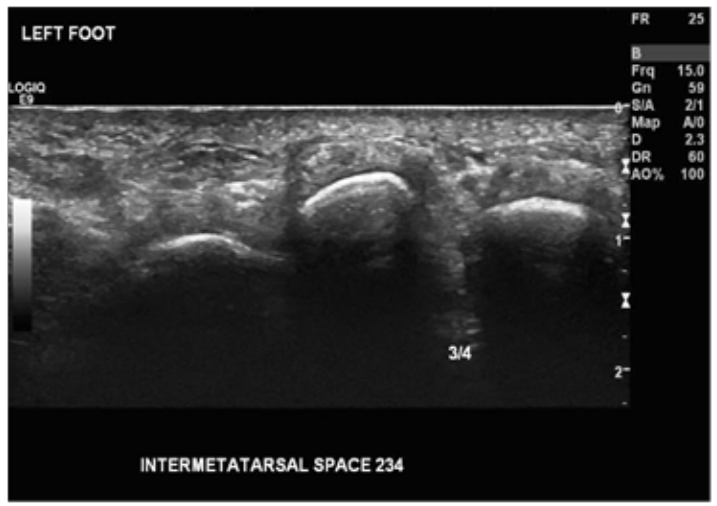

Figure 13 US image shows a chronic metatarsalgia.

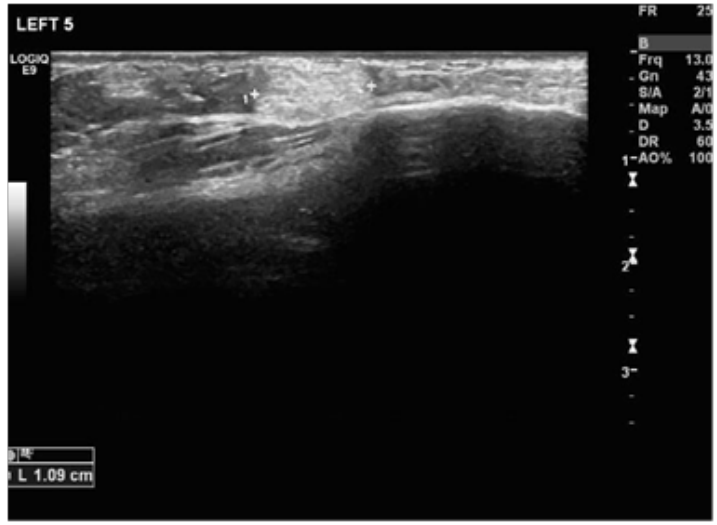

Figure 14 US image shows a lipoma.

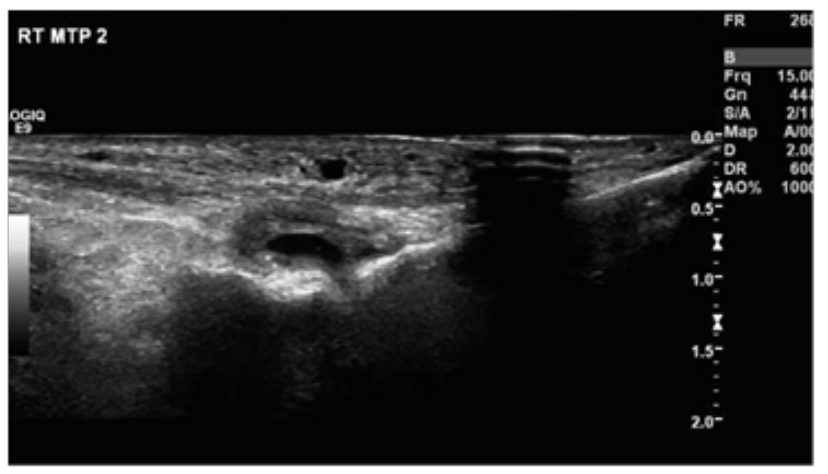

Figure I 5 US image shows a MTP joint effusion and synovitis.

\section{Conclusion}

To improve the achievement of a correct diagnosis of forefoot/nonspecific foot pain, healthcare professionals need to take a complete and careful clinical history and perform thorough physical examinations. This will avoid unnecessary visits to GPs, orthopaedic surgeons and radiologists. Even during an imaging evaluation Morton's neuroma maybe wrongly diagnosed leading to unnecessary and wrong treatments. Therefore it is highly reccommended that radiologists carefully scan the webspaces and have a clear understanding of the various differentials to Morton's neuroma. This will result in a better quality of care and reduce the number of repeated patient visits.

The awareness and understanding of other differential diagnoses is of paramount importance as we have realized that Morton's neuroma diagnosis was present in only $22 \%$ of cases in our series (with that clinical suspicion for the scan request) suggesting that there is a poor understanding of these differential diagnoses among the referrers.

We have tried to convey the importance of lateral thinking that not every forefoot problem is a Morton's neuroma and all other diagnosis should be contemplated, if Morton's neuroma is not seen sonographically, in order to explain patient's symptoms. Hence in our practice we scan the whole foot with an open mind, in order to solve the clinical problem and help in our patient care with effective and correct diagnoses.

\section{Acknowledgements}

None.

\section{Conflict of interest}

The authors declare that there are no conflict of interests involved in this study.

\section{References}

1. Ahn JM, El-Khoury GY. Radiologic evaluation of chronic foot pain. Am Fam Physician. 2007;76(7):975-983.

2. Ashman CJ, Klecker RJ, Yu JS. Forefoot pain involving the metatarsal region: Differential diagnosis with MR imaging 1. Radiographics. 2001;21(6):1425-1440.

3. Coughlin MJ. Common causes of pain in the forefoot in adults. $J$ Bone Joint Surg Br. 2000;82(6):781-790.

4. Morton TG. A peculiar and painful affection of the fourth metatarsophalangeal articulation. Am J Med Sci. 1876;71(141):37-45.

5. Thomas JL, Blitch EL, Chaney DM, et al. Diagnosis and treatment of forefoot disorders. section 3. morton's intermetatarsal neuroma. J Foot Ankle Surg. 2009;48(2):251-256.

Citation: Mehta A, Chourasia A, Urigo C, et al.All forefoot's pain are not morton's neuroma-a pictorial review of the forefoot musculoskeletal conditions mimicking mortons neuroma. Int J Radiol Radiat Ther. 2018;5(5):280-284. DOI: I0.15406/ijrrt.2018.05.00I79 
6. Gregg J, Marks P. Metatarsalgia: An ultrasound perspective. Australas Radiol. 2007;51(6):493-499.

7. Musson RE, Sawhney JS, Lamb L, et al. Ultrasound guided alcohol ablation of morton's neuroma. Foot Ankle Int. 2012;33(3):196-201.

8. Gregg J, Silberstein M, Schneider T, et al. Sonographic and MRI evaluation of the plantar plate: A prospective study. Eur Radiol. 2006;16(12):26612669
9. Callegari L, Leonardi A, Bini A, et al. Ultrasound-guided removal of foreign bodies: Personal experience. Eur Radiol. 2009;19(5):1273-1279.

10. Banal F, Gandjbakhch F, Foltz V, et al. Sensitivity and specificity of ultrasonography in early diagnosis of metatarsal bone stress fractures: A pilot study of 37 patients. J Rheumatol. 2009;36(8):1715-1719. 